\title{
Vorwort des Editor in Chief
}

\section{Gerhard Mayer}

Montanuniversität Leoben, Leoben, Österreich

Online publiziert 3. April 2018

\section{Sehr geehrte Leserinnen und Leser!}

Wie im vorausgegangenen Heft bereits angekündigt, finden sich in der vorliegenden Ausgabe der Berg- und Hüttenmännischen Monatshefte Beiträge, die an die zuvor behandelte Thematik anschließen.

Im Heft 02/2018 wurde ein internationales Forschungsprojekt im Überblick vorgestellt durch Ouchterlony et al. "Sustainable Low Impact Mining - SLIM." Arbeitspakete dieses Projektes, die wesentlich von der Montanuniversität Leoben mitgetragen werden, finden sich in den beiden nachstehend beschriebenen Beiträgen.

Die Originalarbeit "UAVs zur Qualitätssteuerung im Tagbau - das Arbeitspaket 5.1 im europäischen Horizon 2020 Forschungsprojekt SLIM“ der Autorengruppe Alexander Tscharf, Gerhard Mayer, Friedrich Fraundorfer, Andreas Gaich beschreibt den wissenschaftlichen Ansatz, mittels UAV Luftbildern aus verschiedenen Blickrichtungen und Aufnahmehöhen die photogrammetrische Aussagequalität über die Kornzusammensetzung des Hauwerkes entscheidend zu verbessern.

Die Autoren Thomas Seidl, Christian Heiss, Maximilian Föger berichten mit ihrem Beitrag unter dem Titel "Entwicklung eines Systems zur Auskleidung von Sprengbohrlöchern beim Einsatz von losem Emulsiossprengstoff" über einen der sprengtechnischen Kernbereiche von SLIM.

Die nachfolgenden Beiträge wurden auf Basis von Vorträgen beim Österreichischen Bergbautag 2017 in Bad Ischl verfasst. Beide widmen sich dem Thema der Automatisierung im Bergbau.

Mark Ganster berichtet in seiner Arbeit zum Thema "Teilautomatisierung sprengtechnischer Abläufe Ober- und Untertage"zum Themenschwerpunkt der Automatisierung im Bergbau, in diesem Fall speziell zur Sprengtechnik.

Andreas Gaich und Markus Pötsch beschreiben in ihrer Arbeit mit dem Titel "3D Bilder und ihre Anwendung im Tagbau" die immer umfangreicher werdenden Nutzungsmöglichkeiten von 3D Bildern im obertägigen Bergbau.

Mit freundlichem Glückauf!

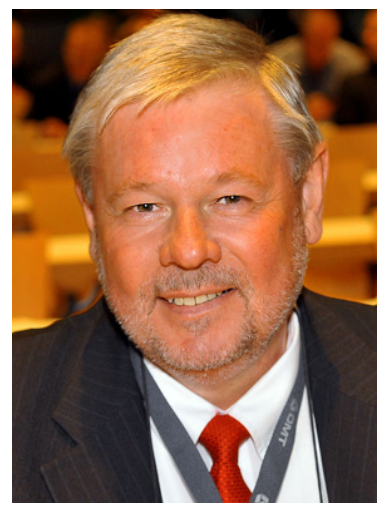

Gerhard Mayer 